\title{
EFECTO DEL NIM (Azadirachta indica JUSS.) SOBRE Bemisia tabaci GENNADIUS (HEMIPTERA: AlEYRODIDAE) Y CONTROLADORES BIOLÓGICOS EN EL CULTIVO DEL MELÓN Cucumis melo L.
}

\author{
NeEM (Azadirachta indica JUSS.) EFFECT ON Bemisia tabaci GENNADIUS \\ (Hemiptera: Aleyrodidae) AND ITS BiOlogiCAl CONTROLlERS IN MELON \\ CROP Cucumis melo L.
}

\section{Bernardo Navarrete*, Oswaldo Valarezo, Ernesto Cañarte y Ramón Solórzano}

Instituto Nacional de Investigaciones Agropecuarias. Departamento Nacional de Protección Vegetal-Entomología. km 12 Vía Santa Ana, Cantón Portoviejo, Manabí, Ecuador. Tel: 052 420 217- 104

*Autor para correspondencia: jose.navarrete@iniap.gob.ec

Manuscrito recibido el 12 de mayo de 2016. Revisado el 8 de junio de 2016. Aceptado el 8 de diciembre de 2016. Publicado el 30 de diciembre de 2016.

\begin{abstract}
Resumen
Esta investigación se realizó en Lodana, cantón Santa Ana, provincia de Manabí, durante los meses de octubre a diciembre del 2005, a nivel de campo e invernadero. Se estudió el efecto de derivados del árbol de nim sobre las poblaciones de la mosca blanca Bemisia tabaci y sus controladores biológicos, además del efecto disuasivo de estas sustancias en la oviposición de B. tabaci en el cultivo de melón. Se utilizó un diseño de bloques completos al azar en arreglo grupal, con cuatro repeticiones. Los tratamientos estudiados fueron extracto acuoso de semillas de nim en dosis de 25; 50; 75 y $100 \mathrm{~g} \mathrm{~L}^{-1}$ y aceite formulado de nim (INBIO-75ß) en dosis de 2,50; 5,00; 7,50 y 10,00 mL L-1. Además se incluyó un testigo químico (imidacloprid $1 \mathrm{~mL} \mathrm{~L}^{-1}$ ) y un testigo con aplicaciones de agua. Se determinó, que de los extractos acuosos, la dosis de $100 \mathrm{~g} \mathrm{~L}^{-1}$ fue el tratamiento más eficaz, sólo superado por el testigo químico en el control de ninfas y adultos de B. tabaci. Los compuestos de nim no tuvieron influencia sobre las poblaciones de depredadores. En el caso de los parasitoides, su población fue menor en las parcelas tratadas con nim. El testigo químico, fue el tratamiento que obtuvo el mejor rendimiento, mientras los tratamientos con nim tuvieron rendimientos superiores al testigo con agua. La prueba de invernadero sugiere un efecto disuasivo de los derivados del nim sobre la oviposición.
\end{abstract}

Palabras claves: insecticidas botánicos, cucurbitáceas, control biológico, moscas blancas. 


\begin{abstract}
This research was carried out in Lodana, Santa Ana, Manabí from October to December of 2005, in order to determine the effect of neem compounds on the populations of Bemisia tabaci and their biological controllers in melon crop. It used a completely randomized block design whit a group arrangement with four repetitions. The treatments had aqueous extract from neem seeds in dosages of 25, 50, 75 and $100 \mathrm{~g} \mathrm{~L}^{-1}$ and formulated oil of neem seeds (INBIO$75(\mathbb{R})$ in dosages of 2,$50 ; 5,00 ; 7,50$ and $10,00 \mathrm{~mL} \mathrm{~L}^{-1}$; besides a chemical witness (Imidacloprid $1 \mathrm{~mL} \mathrm{~L}^{-1}$ ) and an absolute witness (applications of water) were included. Both the aqueous extract and the formulated oil, reduced the populations of eggs, nymphs and adults of $B$. tabaci, being the aqueous extract in dosage of $100 \mathrm{~g} \mathrm{~L}^{-1}$ the most effective treatment, although it was overcome by the chemical witness in the nymphs and adults control. With regard to the effect on the natural enemies, the neem compounds did not have influence on the predatory populations, in the case of parasitoids, their population was minor in the plots with neem. The chemical witness was the treatment that obtained the best result however the treatments with neem had superior yields than the witness treatment. The results of the test carried out in greenhouse, suggest the dissuasive effect of neem compounds on the oviposition of $B$. tabaci.
\end{abstract}

Keywords: botanical insecticides, cucurbits, biological control, whiteflies.

Forma sugerida de citar: Navarrete, Bernardo, Oswaldo Valarezo, Ernesto Cañarte y Ramón Solórzano. 2017. Efecto del nim (Azadirachta indica Juss.) sobre Bemisia tabaci Gennadius (Hemiptera: Aleyrodidae) y controladores biológicos en el cultivo del melón Cucumis melo L. La Granja: Revista de Ciencias de la Vida. Vol. 25(1):33-44. pISSN:1390-3799; eISSN:1390-8596. 


\section{Introducción}

El cultivo de melón Cucumis melo L. es una actividad económica importante en Ecuador, sembrándose, aproximadamente 1.107 ha, con una producción de 7.152 Tm (INEC, 2002). De esta superficie, en Manabí se cultivaron 663 ha, con rendimiento promedio de 11,4 $\mathrm{T} \mathrm{ha}^{-1}$ (MAGAP, 2010). Sin embargo, su producción enfrenta una serie de problemas que reduce el área sembrada y su productividad.

Una limitante de la producción de melón, son los problemas fitosanitarios, destacándose el daño ocasionado por insectos-plaga, situación que se agravó a partir de 1993, con la presencia del biotipo B de "mosca blanca" Bemisia tabaci, Genn. mucho más agresivo y con mayor número de hospederos (Cardona et al., 2005). Los daños provocados por este hemíptero, pueden ser directos por la succión de savia que realizan los estados inmaduros y adultos, e indirectos ocasionados por la proliferación del hongo Capnodium sp., que crece sobre las excreciones azucaradas producidas por las ninfas de mosca blanca, convirtiéndose en fumagina, capaz de interferir en la actividad fotosintética de la planta de melón (Sponagel, 1999). Investigaciones realizadas en Ecuador, demuestran que el melón y otras cucurbitáceas, son preferidas por B. tabaci para ovipositar (Valarezo et al., 2008b). Otro agravante es que el cultivo se realiza en la época seca que coincide con el período de mayor población de este fitófago (INIAP, 1994, 1995).

El control químico, sigue siendo el principal método de manejo por parte del productor, llegando a realizarse más de diez aplicaciones por ciclo de cultivo, empleando productos de elevada toxicidad (Valarezo et al., 2008b). Este uso indiscriminado de plaguicidas trae como consecuencia una serie de problemas, como riesgos para la salud de aplicadores y consumidores, contaminación ambiental y eliminación de controladores biológicos (Mendoza et al., 1995). Este último punto es de gran importancia, ya que se ha demostrado la existencia de parasitoides y depredadores de la plaga, ejerciendo su acción reguladora en melón (Valarezo et al., 2004).

Una alternativa al uso de insecticidas organosintéticos, con menores riesgos al ambiente y la salud pública, es el empleo de extractos acuosos y aceites derivados del árbol del nim Azadirachta indica Juss. (Valarezo et al., 2008a). Al menos 135 metabolitos secundarios han sido hallados en el nim, éstos pueden clasificarse en dos grupos, los isoprenoi- des que incluyen las protomeliacinas, limonoides, azadirone, genudin, vilarin y csecomeliacinas como nimbin, salannin y azadirachtin (Girish y Shankara, 2008) y el otro grupo lo integran ácidos fenólicos como tánnico, gállico, ferúlico y chlorogénico (Singh et al., 2005). Detalles estructurales de estos metabolitos pueden consultarse en la base de datos NeeMDB (Hatti et al., 2014). Estos compuestos han probado su eficacia contra al menos 106 especies de insectos-plaga (Cañarte, 2002). Una de las ventajas del uso del nim en el control de plagas, es que posee varios mecanismos de acción como regulador del crecimiento, antialimentario, repelente, antiovipositor, reductor de la fecundidad e interruptor de la comunicación sexual (Cañarte, 2001; Valarezo et al., 2008a). Estudios recientes realizados a nivel molecular sugieren que el nim afecta la expresión de genes relacionados con protección contra el stress oxidativo en B. tabaci (Asaduzzaman et al., 2015).

Por otro lado, algunos estudios como el realizado por Simmons y Abd-Rabou (2011) y Mohamed et al., (2014) sobre el uso del nim, demuestran que estas sustancias, no afectan a los controladores biológicos, ya que las mismas, deben ser ingeridas para poder actuar. Sin embargo, es de suponer que artrópodos parasíticos, que absorban gran cantidad de azadirachtina de sus víctimas, serán afectados por el nim (Schmutterer, 1990). Al respecto Feldhege y Schmutterer (1993), estudiaron la influencia de las aplicaciones de nim sobre la emergencia y capacidad parasítica de Encarsia formosa sobre Trialeurodes vaporariorum, demostrándose una afectación significativa sobre la emergencia y capacidad parasítica de este benéfico en dosis elevadas de nim. En ese sentido Cóndor (2007) presenta una lista de 13 parasitoides que han sido reportados por la literatura científica como afectados por el nim e indica que las dosis empleadas por los productores, generalmente no tienen un efecto negativo sobre estos controladores y sugiere estudios de campo para comprobar esta hipótesis.

Además, las nuevas tendencias en la producción de alimentos a nivel mundial, están encaminadas a lograr que los consumidores accedan a productos libres de sustancias contaminantes; en este contexto, el uso de derivados del nim, se presenta como una alternativa al empleo de insecticidas sintéticos. Por ello, es notorio el interés en estudiar estas sustancias, como fuente de insecticidas naturales, observando su efecto en los agroecosistemas.

En experimentos realizados por Azevedo et al., 
(2005), Neri et al., (2006), Bleicher et al., (2007) y Zúñiga et al., (2015) se demuestra la eficiencia de los derivados del nim sobre las poblaciones de mosca blanca en melón, haciéndose necesario establecer en la presente investigación la eficacia del extracto acuoso y el aceite formulado, así como su efecto sobre los controladores biológicos presente en este cultivo.

\section{Materiales y métodos}

\subsection{Experimento de campo}

El presente estudio se realizó en Lodana, del cantón Santa Ana, provincia de Manabí, (1 $1^{\circ} 11^{\prime} \mathrm{LS}$, $80^{\circ} 24^{\prime} \mathrm{LO}$ ), durante el período octubre a diciembre del 2005. Se evaluó el efecto sobre B. tabaci y sus controladores biológicos de cuatro dosis de extracto acuoso de semillas de nim (25; 50: 75 y 100 $\left.\mathrm{g} \mathrm{L}^{-1}\right)$ y aceite formulado de nim INBIO-75 $(2)(2,50$; 5,0; 7,5 y $10 \mathrm{~mL} \mathrm{~L}^{-1}$ ), incluyéndose, un testigo químico Confidor $\left(\right.$ (imidacloprid $1 \mathrm{~mL} / \mathrm{L}^{-1}$ ) y un testigo donde se aplicó agua. Se realizaron cinco aspersiones foliares de los tratamientos $(12,21,27,32$ y 42 días después del trasplante -ddt-). Para la siembra se utilizó el híbrido de melón Pacstarß. La parcela experimental fue de $30 \mathrm{~m}^{2}(6 \mathrm{~m} \times 5 \mathrm{~m})$, y el área útil de $15 \mathrm{~m}^{2}(6 \mathrm{~m} \times 2,5 \mathrm{~m})$.

\subsubsection{Levantamiento de la información}

Número de adultos de Bemisia tabaci por hoja. A partir de los 11 días ddt, se registró el número de adultos presentes en el envés de cinco hojas jóvenes, escogidas al azar dentro de plantas en la parcela útil. Esta evaluación se realizó un día antes y uno, dos y tres días después de cada aplicación. En total se realizaron 19 evaluaciones de esta variable.

Estados inmaduros de Bemisia tabaci. A los 30, 37 y $43 \mathrm{ddt}$, se contabilizó con ayuda de un estereomicroscopio, el número de huevos y ninfas vivas de B. tabaci $/ \mathrm{cm}^{2}$. Para esto, se tomaron cinco hojas al azar de la parte basal de la planta, ubicadas en el área útil de cada parcela. Estas fueron llevadas en bolsas plásticas al laboratorio, con papel absorbente en su interior. Las observaciones, se realizaron en dos secciones de hoja de $1 \mathrm{~cm}^{2}$ tomados de la parte basal y apical del envés de cada hoja.
Eficacia de los tratamientos. Se calculó aplicando la fórmula de Abbott (1925), (Ecuación 1).

$$
\% E=\frac{P T e-P T r a t}{P T e} \times 100
$$

Donde:

$\% \mathrm{E}=$ Porcentaje de eficacia, PTe $=$ Población en el testigo, PTrat $=$ Población en el tratamiento.

La población de los tratamientos se calculó empleando el valor del área bajo la curva, mediante la fórmula de Shannon y Finney (Ecuación 2), citado por Juarez et al., (2001):

$$
A B C=\sum_{i=1}^{n}\left[\frac{Y_{i}+Y_{i+1}}{2} \cdot\left(t_{i+1}-t_{i}\right)\right]
$$

Donde:

$A B C=$ Área bajo la curva,

$Y_{i}=$ Población en la i-ésima observación,

$t_{i}=$ Tiempo (días) en la i-ésima observación,

$n=$ número de observaciones.

En este valor se resumen 19 evaluaciones de adultos y tres de inmaduros de B. tabaci.

Número de depredadores por planta. A los 16, 23, 37 y 44 ddt, mediante observación directa se contabilizó el número de depredadores (inmaduros y adultos), presentes en cuatro plantas escogidas al azar dentro de la parcela útil.

Número de ninfas de Bemisia tabaci parasitadas por hoja. Se escogieron aleatoriamente cuatro hojas de la parte basal de plantas dentro del área útil de la parcela, contabilizando en cada hoja, el número de ninfas con evidencia de parasitismo, empleando un estereomicroscopio. Se registró esta variable a los 38 y $46 \mathrm{ddt}$. Las hojas con ninfas parasitadas fueron cortadas en discos que se colocaron sobre cinta adhesiva tipo doble faz pegada sobre cartulina, luego se colocaron en cajas petri, para esperar la emergencia de los parasitoides (Valarezo et al., 2004). Los insectos recuperados fueron ubicados en alcohol al $70 \%$ e identificados a nivel de género.

\subsection{Experimento en invernadero}

Para evaluar el efecto del nim como disuasivo de la oviposición de B. tabaci, se sembraron 18 plantas de 
melón en macetas, aisladas en jaulas de malla hasta tener dos hojas verdaderas, siendo separadas en grupos de tres plantas. Cada grupo de plantas, fue asperjado por el haz y el envés de las hojas, usando un microaspersor, con uno de los siguientes tratamientos: Extracto acuoso de semillas de nim $(50 \mathrm{y}$ $\left.100 \mathrm{~g} \mathrm{~L}^{-1}\right)$, Aceite formulado de nim INBIO-75 (5,0 y $\left.10,0 \mathrm{~mL} \mathrm{~L}^{-1}\right)$, más un testigo químico imidacloprid $\left(1 \mathrm{~mL} \mathrm{~L}^{-1}\right)$ y un testigo absoluto (agua).

Treinta minutos después de su aplicación, las plantas tratadas, fueron trasladadas al campo, a libre exposición, donde permanecieron hasta los cinco días después de la aspersión (dda), con la finalidad de que sean colonizadas por B. tabaci. Para determinar el efecto disuasivo de la oviposición, a los 2 y $5 \mathrm{dda}$, con ayuda de un estereomicroscopio, se contabilizó el número de huevos de B. tabaci por hoja.

\subsection{Rendimiento}

Cuando los frutos llegaron a su madurez, se registraron las siguientes variables: Número de frutos de tamaño comercial y peso del total de frutos cosechados en el área útil, que fueron posteriormente transformados a $\mathrm{kg} \mathrm{ha}^{-1}$.

\subsection{Análisis estadístico}

Para el experimento de campo, se utilizó un diseño de bloques completos al azar en arreglo grupal con cuatro repeticiones. Los datos fueron sometidos a análisis de varianza y para la separación de medias, se aplicó la prueba de significación de Tukey $(0,05)$. Para el estudio de invernadero, se empleó estadística no paramétrica, aplicándose la prueba de Kruskall-Wallis con $k$ muestras (Steel y Torrie, 1988). Para los análisis se utilizó el programa estadístico InfoStat versión 1.0 (InfoStat, 2001).

\section{Resultados}

\subsection{Efecto del nim sobre estados biológicos de Bemisia tabaci}

En la Tabla 1, se presentan los valores bajo la curva de las poblaciones de huevos, ninfas y adultos de B. tabaci, estos fueron utilizados para calcular la eficacia. Se encontraron diferencias entre grupos de tratamientos en las tres variables. En huevos, el extracto acuoso, aceite formulado de nim e imidacloprid formaron un solo rango, con valores estadísticamente inferiores al testigo con agua. Para ninfas y adultos, el extracto acuoso y el aceite formulado fueron iguales entre sí, pero diferentes al imidacloprid, que destaca por su menor valor. Sin embargo, los tres tratamientos, se diferenciaron del testigo con agua. No se detectaron diferencias estadísticas entre las dosis de extracto acuoso, ni aceite de nim en las variables estudiadas, con excepción de la población de ninfas, donde las dosis de 100 y $75 \mathrm{~g}$ $\mathrm{L}^{-1}$ de extracto acuoso, se diferenciaron significativamente con el menor valor.

Se observó una mayor eficacia en el control de huevos de B. tabaci, con el extracto acuoso de nim, en dosis de $100 \mathrm{~g} \mathrm{~L}^{-1}(75 \%)$ y $75 \mathrm{~g} \mathrm{~L}^{-1}(67 \%)$, diferenciándose estadísticamente del resto de tratamientos, siendo el aceite formulado $2,5 \mathrm{~mL} \mathrm{~L}^{-1}$, el que presentó la menor eficacia (29\%). Imidacloprid fue la sustancia con mayor eficacia en el control de ninfas de B. tabaci $(75 \%)$, mientras que el extracto acuoso $25 \mathrm{~g} \mathrm{~L}^{-1}$ y el aceite formulado $7,5 \mathrm{ml} \mathrm{L}^{-1}$, alcanzaron la menor eficacia con $46 \%$ y $47 \%$, respectivamente. Para adultos, nuevamente destaca por su eficacia imidacloprid (44\%), compartiendo rango estadístico con la dosis de extracto acuoso en dosis de $100 \mathrm{~g} \mathrm{~L}^{-1}(35 \%)$.

\subsection{Efecto del nim sobre controladores bio- lógicos}

No fueron observadas diferencias estadísticas en el número de depredadores/planta entre los tratamientos, en ninguna de las cuatro fechas de evaluación. En la Figura 1, se aprecia la distribución porcentual de los principales depredadores asociados a B. tabaci en melón, determinándose, que Zelus spp. (Hemiptera: Reduviidae), fluctuó entre $63 \%$ de individuos registrados en el tratamiento imidacloprid y $32 \%$ en aceite de nim. Chrysopa sp. (Neuroptera: Chrysopidae), fluctuó entre $42 \%$ en extracto acuoso de nim y $17 \%$ en Imidacloprid. Arañas y coleópteros de los géneros Coleomegila y Delphastus alcanzaron porcentajes menores. 
Tabla 1. Valores bajo la curva de las poblaciones de huevos $/ \mathrm{cm}^{2}$, ninfas $/ \mathrm{cm}^{2}$ y adultos/hoja de mosca blanca Bemisia tabaci, obtenidos en los tratamientos para su control en melón. Lodana, 2005.

\begin{tabular}{lccc}
\hline Grupos de Tratamientos & Huevos $/ \mathrm{cm}^{2}$ & Ninfas $/ \mathrm{cm}^{2}$ & Adultos/hoja ${ }^{1}$ \\
\hline Extracto acuoso de semillas de nim & $112,89 a$ & $299,69 b$ & $445,95 b$ \\
Aceite formulado de nim & $186,93 a$ & $338,08 \mathrm{~b}$ & $447,08 \mathrm{~b}$ \\
Imidacloprid $1 \mathrm{~mL} \mathrm{~L}^{-1}$ & $139,93 a$ & $171,73 a$ & $348,95 a$ \\
Testigo absoluto & $354,62 b$ & $701,63 c$ & $628,98 c$ \\
\hline Tukey 0,05 & 87,19 & 94,77 & 47,98 \\
\hline Dosis de extracto acuoso de semillas de nim & & & \\
Extracto acuoso $25 \mathrm{~g} \mathrm{~L}^{-1}$ & 139,7 & $379,98 b$ & 446,4 \\
Extracto acuoso $50 \mathrm{~g} \mathrm{~L}^{-1}$ & 114,94 & $355,30 a b$ & 473,4 \\
Extracto acuoso $75 \mathrm{~g} \mathrm{~L}^{-1}$ & 114,33 & $241,31 a$ & 459,15 \\
Extracto acuoso $100 \mathrm{~g} \mathrm{~L}^{-1}$ & 82,61 & $222,18 a$ & 404,85 \\
\hline Tukey 0,05 & $\mathrm{ns}$ & 134,02 & $\mathrm{~ns}$ \\
\hline Dosis de aceite formulado de nim & & & 445 \\
Aceite formulado $2,50 \mathrm{~mL} \mathrm{~L}^{-1}$ & 217,88 & 352,94 & 440,48 \\
Aceite formulado $5,00 \mathrm{~mL} \mathrm{~L}^{-1}$ & 162,98 & 324,54 & 469,18 \\
Aceite formulado $7,50 \mathrm{~mL} \mathrm{~L}^{-1}$ & 193,74 & 370,16 & 433,68 \\
Aceite formulado $10,00 \mathrm{~mL} \mathrm{~L}^{-1}$ & 173,13 & 304,68 & $\mathrm{~ns}$ \\
Tukey 0,05 & $\mathrm{ns}$ & $\mathrm{ns}$ & 8,23 \\
\hline CV\% & 40,19 & 21,6 &
\end{tabular}

${ }^{1}$ Promedios seguidos con la misma letra no difieren entre si según la prueba de Tukey $(\mathrm{P} \leq 0,05)$

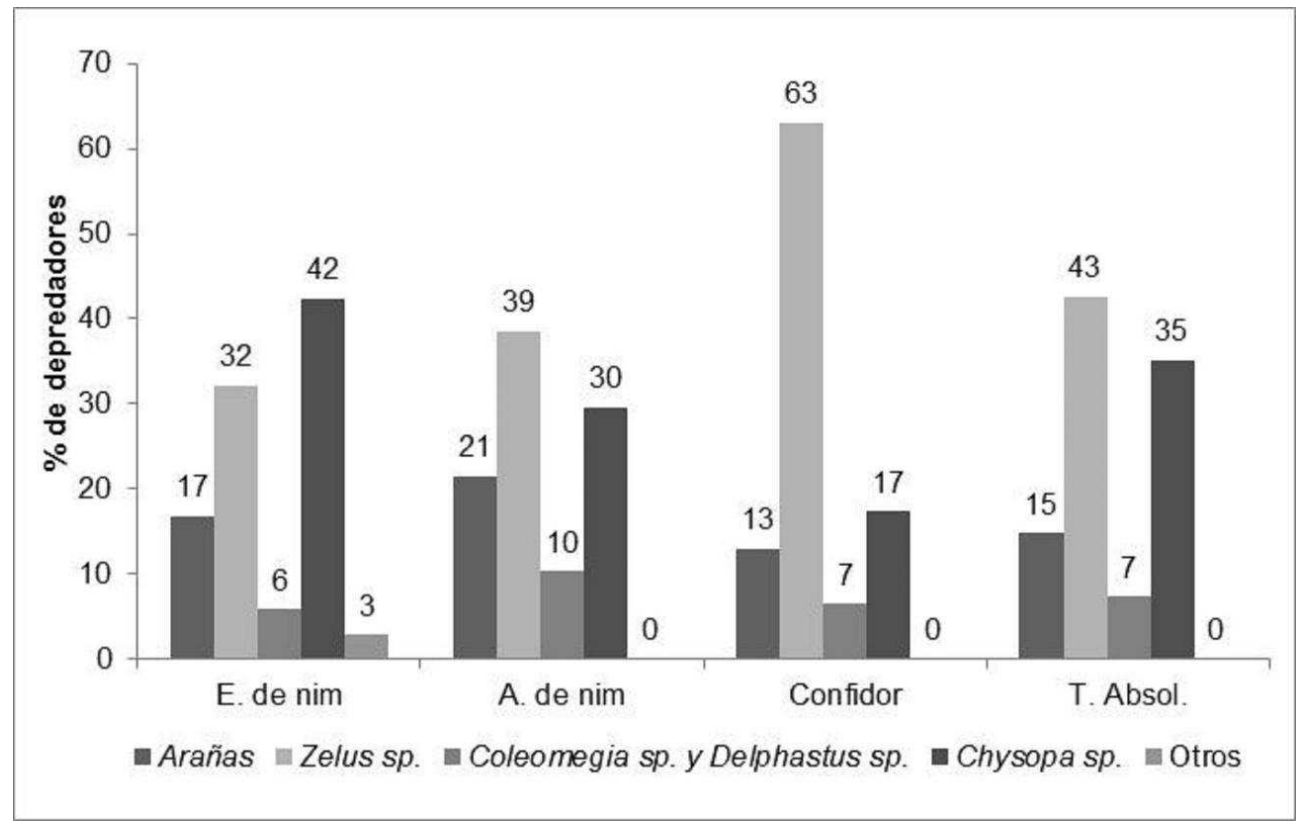

Figura 1. Relación porcentaje de depredadores reportados en los grupos de tratamientos para el control de Bemisia tabaci en melón. 
Tabla 2. Valores promedio de número de ninfas parasitadas/hoja registrado en dos fechas de evaluación en los tratamientos para el control de Bemisia tabaci en melón.

\begin{tabular}{|c|c|c|}
\hline \multirow{3}{*}{ Grupos de Tratamientos } & \multicolumn{2}{|c|}{ No. ninfas parasitadas/hoja } \\
\hline & \multicolumn{2}{|c|}{ Fecha de evaluación } \\
\hline & $01-12-2005^{1,2}$ & 09-12-2005 $5^{1,2}$ \\
\hline Extracto acuoso de semillas de nim & $1,05 a$ & $3,01 a$ \\
\hline Aceite formulado de nim & $3,17 a$ & $6,89 a b$ \\
\hline Imidacloprid $1 \mathrm{~mL} \mathrm{~L}^{-1}$ & $0,88 a$ & $4,50 a$ \\
\hline Testigo absoluto & $11,17 b$ & $10,69 b$ \\
\hline Tukey 0,05 & 0,68 & 0,98 \\
\hline \multicolumn{3}{|c|}{ Dosis de extracto acuoso de semillas de nim } \\
\hline Extracto acuoso $25 \mathrm{~g} \mathrm{~L}^{-1}$ & 1,63 & 3,88 \\
\hline Extracto acuoso $50 \mathrm{~g} \mathrm{~L}^{-1}$ & 1,54 & 1,78 \\
\hline Extracto acuoso $75 \mathrm{~g} \mathrm{~L}^{-1}$ & 0,79 & 3,00 \\
\hline Extracto acuoso $100 \mathrm{~g} \mathrm{~L}^{-1}$ & 0,25 & 3,38 \\
\hline Tukey 0,05 & $\mathrm{~ns}$ & $\mathrm{~ns}$ \\
\hline \multicolumn{3}{|l|}{ Dosis de aceite formulado de nim } \\
\hline Aceite formulado $2,50 \mathrm{~mL} \mathrm{~L}^{-1}$ & 3,88 & 7,06 \\
\hline Aceite formulado $5,00 \mathrm{~mL} \mathrm{~L}^{-1}$ & 5,38 & 9,81 \\
\hline Aceite formulado $7,50 \mathrm{~mL} \mathrm{~L}^{-1}$ & 1,13 & 5,11 \\
\hline Aceite formulado $10,00 \mathrm{~mL} \mathrm{~L}^{-1}$ & 2,31 & 5,56 \\
\hline Tukey 0,05 & ns & $\mathrm{ns}$ \\
\hline CV\% & 29,43 & 32,19 \\
\hline
\end{tabular}

Cuando se evaluó el efecto del nim sobre el parasitismo de $B$. tabaci, se determinaron diferencias estadísticas entre grupos de tratamientos, en las dos fechas de evaluación, observándose, que el nim, sea como extracto acuoso o aceite y el imidacloprid, presentaron valores significativamente menores de ninfas de B. tabaci parasitadas, comparado con el testigo con agua. Por otro lado, no se reportaron diferencias estadísticas entre las dosis de extracto acuoso y aceite de nim para la variable ninfas parasitadas (Tabla 2). Durante esta investigación, fueron identificados los parasitoides Encarsia sp. y Eretmocerus sp. (Hymenoptera: Aphelinidae), ambos eficientes reguladores de $B$. tabaci.

\subsection{Efecto del nim como disuasivo de la ovi- posición de Bemisia tabaci}

Al analizar la información sobre el efecto del nim como disuasivo de la oviposición de $B$. tabaci a nivel de invernadero, no se registró diferencias estadísticas entre tratamientos a los 2 dda. No obstante, a los 5 dda se marcaron diferencias estadísticas entre tratamientos, estableciéndose tres rangos de significación. En los tratamientos con nim la población de huevos/hoja fue significativamente más baja que en el testigo con agua, con excepción del aceite formulado en dosis de 5,00 $\mathrm{mL} \mathrm{L}^{-1}$ (Tabla 3). 
Tabla 3. Valores promedio del número de huevos de Bemisia tabaci por hoja, encontrados en los tratamientos para determinar la reducción de oviposición causada por extractos acuosos y aceites formulado de nim en plántulas de melón.

\begin{tabular}{lcc}
\hline \multirow{2}{*}{ Tratamientos } & \multicolumn{2}{c}{ Número de huevos/hoja ${ }^{1}$} \\
\cline { 2 - 3 } & $\mathbf{2}$ dda & $\mathbf{5 ~ d d a}$ \\
\hline Extracto acuoso $50 \mathrm{~g} \mathrm{~L}^{-1}$ & 1 & $5,25 \mathrm{a}$ \\
Extracto acuoso $100 \mathrm{~g} \mathrm{~L}^{-1}$ & 3,75 & $11,67 \mathrm{ab}$ \\
Aceite formulado $5 \mathrm{~mL} \mathrm{~L}^{-1}$ & 2,67 & $26,67 \mathrm{bc}$ \\
Aceite formulado $10 \mathrm{~mL} \mathrm{~L}^{-1}$ & 2 & $15,67 \mathrm{ab}$ \\
Imidacloprid 1 mL L & 1 & $8,33 \mathrm{ab}$ \\
Testigo absoluto & 10,67 & $47 \mathrm{c}$ \\
\hline Kruskall-Wallis (H) & $8,58^{\mathrm{ns}}$ & $14,61^{*}$ \\
\hline Tratamientos con letras iguales no difieren estadísticamente \\
según la prueba no paramétrica de Kruskall y Wallis (P $\leq 0,05)$
\end{tabular}

Tabla 4. Valores promedio de número de frutos, kilogramos/parcela y rendimiento $\mathrm{kg} / \mathrm{ha}$ obtenidos en los tratamientos para el control de Bemisia tabaci en melón.

\begin{tabular}{|c|c|c|c|}
\hline Grupos de Tratamientos & No. frutos/parcela ${ }^{1}$ & kg /parcela ${ }^{1}$ & kg/ha \\
\hline Extracto acuoso de semilla de nim & $24,05 b$ & $14,05 b$ & 10220 \\
\hline Aceite formulado de nim & $24,42 b$ & $13,78 b$ & 10022 \\
\hline Imidacloprid $1 \mathrm{~mL} \mathrm{~L}^{-1}$ & $28,52 a$ & $28,26 a$ & 20555 \\
\hline Testigo absoluto & $17,89 c$ & $7,89 c$ & 5740 \\
\hline Tukey 0,05 & 5,25 & 5,36 & \\
\hline \multicolumn{4}{|c|}{ Dosis de extracto acuoso de semillas de nim } \\
\hline Extracto acuoso $25 \mathrm{~g} \mathrm{~L}^{-1}$ & 22,69 & 13,4 & 9742 \\
\hline Extracto acuoso $50 \mathrm{~g} \mathrm{~L}^{-1}$ & 23,85 & 11,96 & 8699 \\
\hline Extracto acuoso $75 \mathrm{~g} \mathrm{~L}^{-1}$ & 23,49 & 13,44 & 9775 \\
\hline Extracto acuoso $100 \mathrm{~g} \mathrm{~L}^{-1}$ & 26,16 & 17,41 & 12664 \\
\hline Tukey 0,05 & ns & ns & \\
\hline \multicolumn{4}{|l|}{ Dosis de aceite formulado de nim } \\
\hline Aceite formulado $2,50 \mathrm{~mL} \mathrm{~L}^{-1}$ & 22,75 & 12,08 & 8784 \\
\hline Aceite formulado $5,00 \mathrm{~mL} \mathrm{~L}^{-1}$ & 25,07 & 13,03 & 9474 \\
\hline Aceite formulado $7,50 \mathrm{~mL} \mathrm{~L}^{-1}$ & 25,58 & 14,16 & 10299 \\
\hline Aceite formulado $10,00 \mathrm{~mL} \mathrm{~L}^{-1}$ & 24,3 & 15,85 & 11530 \\
\hline Tukey 0,05 & ns & ns & \\
\hline $\mathrm{CV} \%$ & 16,89 & 28,37 & \\
\hline
\end{tabular}




\subsection{Rendimiento}

Se observaron diferencias estadísticas entre grupos de tratamientos para las variables; número de frutos/parcela y peso en $\mathrm{kg} /$ parcela. En ambas se destacó significativamente el testigo químico (imidacloprid). El nim, extracto acuoso o aceite formulado, formó un segundo rango de significación, con valores inferiores al testigo químico, pero estadísticamente superior al testigo con agua (Tabla 4). Las dosis de extracto acuoso y aceite de nim, fueron estadísticamente iguales entre sí.

\section{Discusión y conclusiones}

Fue evidenciada la reducción de las poblaciones adultas de $B$. tabaci en aquellas parcelas tratadas con nim en extracto acuoso y aceite, confirmándose la actividad repelente de estas sustancias, lo cual según Cañarte (2002), es atribuible a la presencia de volátiles en el nim, que son liberados como mensajes olfatorios, que evitan que los insectos colonicen el área tratada. La aplicación de nim, tuvo un efecto reductor sobre los estados inmaduros de $B$. tabaci, explicable por la disminución de adultos y el efecto disuasivo del nim sobre la oviposición de esta plaga, lo cual es corroborado con estudios realizados por Prabhaker et al. (1999).

Adicionalmente, hay un efecto sobre la población de ninfas, que se ve reducida, como consecuencia del menor número de adultos y huevos en las plantas tratadas. Es posible que la disminución de ninfas, esté asociada también al efecto directo de la azadirachtina sobre los estados inmaduros de $B$. tabaci, lo que es coincidente con Prabhaker et al., (1999), El Shafie (2001), Ktattack et al. (2001), Silva et al. (2003), Azevedo et al. (2005).

La falta de significación estadística en la población de depredadores entre tratamientos, sugiere, que los compuestos a base de nim, además del imidacloprid, no afectaron la actividad de estos controladores biológicos en melón, posiblemente por su acción sistémica, o por el hecho que sustancias a base de nim, tendrían que ser ingerido para poder actuar. Sin embargo, es de suponer que artrópodos benéficos que absorban gran cantidad de azadirachtina de sus víctimas, serán afectados (Schmutterer, 1990). Es importante destacar, que estos mismos grupos de depredadores, han sido reportados como importantes reguladores de las poblaciones de $B$. $t a$ baci en Ecuador (INIAP, 1996; Valarezo et al., 2008b).
Se determinó un efecto negativo del nim sobre los parasitoides, que pudiera interferir en el encuentro de hospedero por parte del parasitoide, o ejerciendo un efecto repelente sobre estos organismos. En este sentido, en trabajos realizados por Price y Schuster (1991), Feldhege y Schmutterer (1993) y Stansly y Liu (1997), se ha determinado, que bajo ciertas condiciones (época de aplicación, dosis, entre otras), el nim puede afectar a los parasitoides. Es posible que esta reducción de la población de ninfas parasitadas, se deba también a que este factor de regulación, es densodependiente, por lo que es lógico encontrar mayor población de biocontroladores en parcelas con mayor presencia del fitófago, como en el testigo. Mientras que las parcelas tratadas con nim, al presentar menos número de ninfas de $B$. $t a-$ baci, tuvieron igualmente menor parasitismo. Finalmente, se puede mencionar que los parasitoides reportados durante este estudio, coinciden con aquellos encontrados por Valarezo et al., (2004), como reguladores de $B$. tabaci en melón en la provincia de Manabí.

Los resultados obtenidos a nivel de invernadero sugieren que el nim tiene un efecto disuasivo sobre la oviposición de B. tabaci, fenómeno observado por Prabhaker et al., (1999) en algodón. En otros estudios realizados por El-Sinary y Rizk (2002) y Singh y Singh (1998), se demostró el efecto disuasivo de la oviposición del nim sobre insectos-plagas como Pthorimaea opercullela y Bactrocera spp., respectivamente.

La menor población de huevos observada en la dosis de $50 \mathrm{~g} / \mathrm{L}^{-1}$ en comparación la dosis más alta $\left(100 \mathrm{~g} / \mathrm{L}^{-1}\right)$ es un resultado inusual, sin embargo, se aprecia que estadísticamente comparten el mismo rango de significación, por lo que ésta diferencia numérica puede ser atribuida a efectos aleatorios, considerando, que sólo se realizó una réplica biológica.

Los resultados permiten advertir que la reducción de los estados biológicos de B. tabaci por efecto del nim, si influyeron positivamente en el rendimiento del cultivo de melón. Sin embargo, al ser una sustancia natural, no alcanzó a competir con el testigo químico (imidacloprid), que prácticamente duplicó su rendimiento con relación a los tratamientos con nim, lo cual fue influenciado muy posiblemente por la eficacia del químico, en el control de adultos y ninfas de $B$. tabaci, además de su actividad de amplio espectro.

Basados en los resultados de campo de este es- 
tudio, se establecen las siguientes conclusiones:

Las aplicaciones de nim disminuyeron las poblaciones de huevos, ninfas y adultos de B. tabaci en melón.

Los derivados del nim no afectaron las poblaciones de depredadores, pero si interfirieron en la actividad de los parasitoides.

Los tratamientos con nim tuvieron rendimientos superiores al testigo sin aplicaciones.

\section{Referencias}

Abbott, W. 1925. A method of computing the effectiveness of an insecticide. J. Econ. Entomol. 18:265-267.

Asaduzzaman, M., J. K. Shim, S. Lee y K. Y. Lee. 2015. Azadirachtin ingestion is lethal and inhibits expression of ferritin and thioredoxin peroxidase genes of the sweetpotato whitefly Bemisia tabaci. Journal of Asia-Pacific Entomology. 19(1):1-4. DOI:10.1016/j.aspen.2015.10.011

Azevedo, F., J. Guimaraes, R. Braga y M. Lima. 2005. Eficiencia de produtos naturais para o controle de Bemisia tabaci biotipo B (Hemiptera: Aleyrodidae) em meloeiro. Arq. Inst. Biol. 72(1):73-79. http://www.biologico.sp.gov.br/docs/arq/V 72_1/azevedo.PDF

Bleicher, E. M., Gonçalves y L. da Silva. 2007. Efeito de derivados de nim aplicados por pulverização sobre a mosca-branca em meloeiro. Horticultura Brasileira. 25(1):110-113. https://www.researchgate.net/profile/Ervin o_Bleicher/publication/262632013_Effects_of _neem_derivatives_sprayed_on_melon_crop_ to_control_silverleaf_whitefly/links /54b93cff 0cf253b50e2900c0.pdf

Cañarte, E. 2001. El minador de la hoja de los cítricos Phyllocnistis citrella Stainton (Lepidoptera: Gracillaridae) en Ecuador. Tesis de Maestro en Ciencias. Colegio de Postgraduados, Instituto de Fitosanidad, Especialidad de Entomología y Acarología.
Cañarte, E. 2002. El nim Azadirachta indica A. Juss (Meliaceae) y su rol en la agricultura rentable del Ecuador. Memorias XIX Reunión Latinoamericana de maíz. Portoviejo, EC. 1 disco compacto, $8 \mathrm{~mm}$.

Cardona, C., A. López-Ávila y O. Valarezo. 2005. Colombia and Ecuador. Whitefly and whitefly-borne viruses in the tropics: building a knowledge base for global action. Ed. P. Anderson y F. Morales. CIAT, Cali.

Cóndor, A. F. 2007. Effect of neem (Azadirachta indica Juss) insecticides on parasitoids. Rev. peru. biol. 14(1):069-074. http://www.scielo.org.pe/pdf/rpb/v14n1/v 14n01a16.pdf

El Shafie, A. 2001. The use of neem products for sustainable management of homopterous key pest on potato and eggplant in the Sudan. Thesis of Doctor in Agriculture. University of Giessen. http://geb.uni-giessen.de/geb/volltexte/200 1/516/pdf/d010119.pdf

El-Sinary, N. y A. Rizk. 2002. Oviposition deterrence and other biological influences of aqueous leaves and extracts of neem, colocasia, and their mixtures alone or combine with gamma radiation to reduce the risk of the potato tubermoth, Phthorimae operculella (Zeller). Pakistan Journal of Bioscience. 5(9):911-914. http:/ /198.170.104.138/pjbs/2002/911-914.p $\mathrm{df}$

Feldhege, M. y H. Schmutterer. 1993. Investigations on side-effects of Margosan-O on Encarsia formosa Gah. (Hy, Aphelinidae), parasitoid of the greenhouse whitefly, Trialeurodes vaporariorum Westw. (Hom.; Aleyrodidae). Journal of Appl. Entomology. 115(1):3742. DOI:10.1111/j.1439-0418.1993.tb00361.x

Girish, K. y S. Shankara Bhat. 2008. Neem-a green treasure. Electronic journal of Biology. 4(3):102-111. http://eprints.uni-mysore.ac.in /id/eprint/15031

Hatti, K.S., L. Muralitharan, R. Hegde y A. Kush. 2014. NeeMDB: convenient database for neem secondary metabolites. Bioinformation. 10(5):314. DOI:10.6026/97320630010314 
InfoStat. 2001. Software Estadístico. Versión 1.0. Manual del usuario. Facultad de Ciencias Agropecuarias. Universidad Nacional de Córdoba. Córdoba. https:/ / www.researchgate.net/file.PostFileL oader.html?id=56d87439eeae395144018193\&a ssetKey=AS\%3A335605960593408\%401457026 104675

Instituto Nacional Autónomo de Investigaciones Agropecuarias, I.N.I.A.P. 1994. Informe Técnico Anual. Departamento Nacional de Protección Vegetal., Sección Entomología. Portoviejo, EC., Estación Experimental Portoviejo.

Instituto Nacional Autónomo de Investigaciones Agropecuarias, I.N.I.A.P. 1995. Informe Técnico Anual. Departamento Nacional de Protección Vegetal., Sección Entomología. Portoviejo, EC., Estación Experimental Portoviejo.

Instituto Nacional Autónomo de Investigaciones Agropecuarias, I.N.I.A.P. 1996. Informe Técnico Anual. Departamento Nacional de Protección Vegetal., Sección Entomología. Portoviejo, EC., Estación Experimental Boliche.

Instituto Nacional de Estadísticas y Censos, I.N.E.C. y Ministerio de Agricultura y Ganadería, M.A.G. 2002. III Censo Nacional Agropecuario. Resultados Nacionales y Priovinciales. 1 disco compacto $8 \mathrm{~mm}$.

Juarez, H.S., J. Amaro, M. Rivera, A. Párraga, y R.J. Hijmans. 2001. Efecto del nitrógeno en el Tizón Tardío de la papa. CIP, Lima. https://www.researchgate.net/profile/Henr y_Juarez/publication/249962566_Efecto_del_ Nitrgeno_en_el_Tizn_Tardo_de_la_Papa/link s/00b4951e6f66217fc8000000.pdf

Ktattack, M., L. Khan, M. Awan y A. Husain. 2001. Evaluation of some insecticidal combination and neem (Azadirachta indica A. Juss.) Extracts against jassids and whitefly on cotton and their effect on the yield. Pakistan Journal of Bioscience. 4(4):419-421.

Ministerio de Agricultura Ganadería Acuacultura y Pesca, M.A.G.A.P. 2010. Consolidado de Estadísticas Agropecuarias. MAGAP, Portoviejo.
Mendoza, J., O. Valarezo, M. Arias, R. Quijije, E. Cañarte y V. Álvarez. 1995. Reporte de Ecuador. IV Taller Latinoamericano sobre moscas blancas y geminivirus. Memorias. CEIBA. Honduras, 16-18 de octubre. 36(1):13-15. http://bdigital.zamorano.edu/bitstream/110 36/3667/1/10.pdf

Mohamed ES, H. Abdelgader y A. Satti. 2014. Field evaluation of a newly introduced thiamethoxam insecticide and neem seed water extract against the predator Hippodamia variegata in Sudan. International Journal of Agriculture Innovations and Research. 3(3):931-935. https:/ / www.researchgate.net/profile/Abda lla_Satti/publication/271504234_Field_Evalu ation_of_a_Newly_Introduced_Thiamethoxa m_Insecticide_and_Neem_Seed_Water_Extra ct_Against_the_Predator_Hippodamia_Varie gata_in_Sudan/links/54c913120cf25087c4ec4 d28.pdf

Neri, D., G. de Góis, P. Maracaja, D. de Medeirose y G. Nunes. 2006. Efeito do extrato aquoso de nim sobre Bemisia tabaci biótipo B (Gennadius)(Hemiptera: Aleyrodidae), em meloeiro. Revista Verde de Agroecologia e Desenvolvimento Sustentável. 1(2): 48-53. http://www.gvaa.com.br/revista/index.php /RVADS/article/download/15/15

Prabhaker, N., N. Toscano y T. Henneberry. 1999. Comparison of neem, urea and amitraz as oviposition supressants and larvicides against Bemisia argentifolii (Homoptera: Aleyrodidae). Journal of Economic Entomology. 92(1): 41-45. DOI:http:/ /dx.doi.org/10.1093/jee/92.1.40

Price, J. y D. Schuster. 1991. Effects of natural and synthetic insecticides on sweetpotato whitefly Bemisia tabaci (Homoptera: Aleyrodidae) and its hymenopterus parasitoids. Florida Entomologist. 74(1):60-68. http://www.jstor.org/stable/3495240

Schmutterer, H. 1990. Properties an potencial of natural pesticides from the neem tree. Annun. Rev. Entomol. 35: 271-297. DOI:10.1146/annurev.en.35.010190.001415

Silva, L., E. Bleicher y A. Araujo. 2003. Eficiencia de azadirachtina no controle de mosca branca en meloeiro sob condicoes de vegetacao e de 
campo. Horticultura Brasileira. 21(2): 198-201. http://www.scielo.br/pdf/\%0D/hb/v21n2/ a15v21n2.pdf

Simmons, A. y A. Shaaban. 2011. Populations of predators and parasitoids of Bemisia tabaci (Hemiptera: Aleyrodidae) after the application of eight biorational insecticides in vegetable crops. Pest management science. 67(8):10231028. DOI:10.1002/ps.2155

Singh, S. y P. Singh. 1998. Neem (Azadirachta indica) seed kernel extracts and azadirachtin as oviposition deterrents against the melon fly (Bactrocera cucurbita) and the oriental fruit fly (Bactrocera dorsalis). Phytoparasitica. 26(3): 1-7. DOI:10.1007/BF02981434

Singh, U., S. Maurya y D. Singh. 2005. Phenolic acids in neem (Azadirachta indica) a major pre-existing secondary metabolites. Journal of herbal pharmacotherapy. 5(1): 35-43. DOI:10.1080/J157v05n01_05

Sponagel, K. 1999. Presencia, estatus de peste y manejo de la mosca blanca del algodón (Bemisia tabaci) y de la mosca blanca del invernadero (Trialeurodes vaporariorum). SESA, Quito.

Stansly, P., y T. Liu. 1997. Selectivity of insecticides to Encarsia pergandiella (Hymenoptera: Aphelinidae), an endoparasitoid of Bemisia argentifolii (Hemiptera: Aleyrodidae). Bulletin of Entomological Research. 87: 525-531. http:/ / agris.fao.org/agris-search/search.do? recordID=GB1997025801

Steel, R. y J. Torrie. 1988. Bioestadística. Principios y procedimientos. McGraw-Hill. México. Segunda Edición.

Valarezo, O., E. Cañarte, B. Navarrete, J. Guerrero y B. Arias. 2004. Las moscas blancas en el Ecuador: diagnóstico de su situación, impacto y enemigos naturales. INIAPSESA-PROMSA. Portoviejo. Manual No. 57. http:/ /agris.fao.org/agris-search/search.do? recordID=EC2005000019

Valarezo, O., E. Cañarte y B. Navarrete. 2008a. El nim: insecticida botánico para el manejo de plagas agrícolas. INIAP. Portoviejo. Boletín Divulgativo No. 336. https://www.researchgate.net/publication/3 02044854_El_nim_Insecticida_botanico_para_ el_manejo_de_plagas_agricolas

Valarezo, O., E. Cañarte, B. Navarrete, J. Guerrero y B. Arias. 2008b. Diagnóstico de la mosca blanca en Ecuador. La Granja. 7(1):13-20. http://revistas.ups.edu.ec/index.php/granja /article/view/517/374

Zúniga, B., D. Núñez y L. Marroquín. 2015. Comparación de dos productos botánicos vrs. un sintético para control de mosca blanca en cultivo de melón. Portal de la Ciencia. 4:45-51. DOI:http://dx.doi.org/10.5377/pc.v4i0.1861 